\title{
Initiation of intravitreal aflibercept injection treatment in patients with diabetic macular edema: a review of VIVID-DME and VISTA-DME data
}

Focke Ziemssen ${ }^{1 *}(0)$, Patricio G. Schlottman ${ }^{2}$, Jennifer I. Lim ${ }^{3}$, Hansjürgen Agostini ${ }^{4}$, Gabriele E. Lang ${ }^{5}$ and Francesco Bandello ${ }^{6}$

\begin{abstract}
Background: Diabetic macular edema (DME) shows a gradual and sustained functional and morphologic response to anti-vascular endothelial growth factor (VEGF) drugs, but the optimal schedule for initiation of anti-VEGF therapy is not known. This study evaluates the treatment response behavior of DME in the Phase 3 trials of intravitreal aflibercept, with 5 initial intravitreal aflibercept injections (IAI), 2 mg every 4 weeks (2q4), in the upload phase.

Methods: This post hoc pooled analysis of the VISTA-DME (NCT01363440) and VIVID-DME (NCT01331681) trials evaluated the change in best-corrected visual acuity (BCVA) and central retinal thickness (CRT) during the upload phase, using pooled data from both IAI treatment groups [ $2 \mathrm{q} 4$ and 2 mg every 8 weeks (2q8)]. The mean visit-tovisit change in BCVA and CRT, and the respective rate of gainers and losers was calculated for each successive visit. A secondary analysis compared the visit-to-visit change in BCVA between the $2 q 4$ and $2 q 8$ treatment arms during the upload period and the first year treatment period.

Results: The majority of eyes showed a continuing improvement of BCVA after the first IAI. The proportions of eyes gaining BCVA ( $\geq 5$ letters) at each visit compared with the previous visit during the IAI $2 q 4$ upload phase were 60 (4-weeks), 19 (8-weeks), 16 (12-weeks), 15 (16-weeks), and $14 \%$ (20-weeks). In contrast, the proportions of eyes losing BCVA ( $\geq 5$ letters) were 3 (4-weeks), 7 (8-weeks), 7 (12-weeks), 9 (16-weeks), and $8 \%$ (20-weeks), respectively. The odds of BCVA ( $\geq 5$ letters) gain/loss exceeded 1.7 at each visit (range 1.7-20). Overall, the proportion of patients with BCVA gain $\geq 5$ letters at week 20 (compared with baseline) was 76 and $80 \%$ in the $2 q 4$ and 2 q8 groups, respectively. The proportions of eyes showing a visit-to-visit decrease in CRT of $\geq 30 \mu \mathrm{m}$ during the first 5 IAI were 77 (4-weeks), 27 (8-weeks), 21 (12-weeks), 17 (16-weeks), and $12 \%$ (20-weeks). In the secondary analysis, the BCVA outcomes were similar for the $2 \mathrm{q} 8$ and $2 \mathrm{q} 4$ treatment arms.
\end{abstract}

Conclusions: The data presented here are consistent with continual functional and anatomic improvement following the fourth and fifth initial 2q4 injections, suggesting that an intensive and sufficiently long upload may be beneficial.

Trial registration VIVID-DME: Clinicaltrials.gov: NCT01331681; VISTA-DME: Clinicaltrials.gov: NCT01363440

Keywords: Aflibercept, Anti-VEGF, Diabetic macular edema, Upload, Treatment initiation, Loading dose

\footnotetext{
${ }^{*}$ Correspondence: focke.ziemssen@med.uni-tuebingen.de

${ }^{1}$ Centre for Ophthalmology, Eberhard-Karls University of Tuebingen,

Schleichstrasse 12, 72076 Tübingen, Germany

Full list of author information is available at the end of the article
} 


\section{Background}

Intravitreal anti-vascular endothelial growth factor (VEGF) medications are recognized for improving visual outcomes and decreasing macular fluid in patients with diabetic macular edema (DME) [1-3]. In contrast to other retinal diseases, the response has been shown to be more "gradual" - the curves of best-corrected visual acuity (BCVA) and central retinal thickness (CRT) more slowly approach their values of maximal improvement during the treatment every 4 weeks (Table $1 ;$ [4-12]), resulting in a long first phase of improvement [1]. Importantly, long-term studies have shown that the favorable outcome can be maintained for at least 3-5 years, with a significantly reduced number of treatments during the later follow-up [6].

What is less clear at this point, however, is the optimal dosing schedule for initiation of anti-VEGF therapy in DME. Given that the disease is characterized by high levels of intravitreal VEGF [9], there is clearly a rationale for aggressive initial therapy; however, specific initiation algorithms have not been evaluated as the independent variable within Phase 3 clinical trials, and indirect comparisons-across study populations or drugs usedare of limited value. The 36-month results from the DA VINCI study suggest that 5 initial $2 \mathrm{mg}$ intravitreal aflibercept injections (IAI) may be beneficial, whereas the 2-year Protocol $\mathrm{T}$ results show that individualized initiation schemes that led to more than 5 doses for initiation resulted in a favorable outcome [13, 14]. There are currently no clearly defined predictors for identifying patients with DME who are in need of either an intensified or less intensive initial treatment with anti-VEGF therapy.
A worldwide comparison showed a widespread diversity of initiation recommendations for anti-VEGF treatment in DME, ranging from series of 3,4 , or 5 to patterns of $4+2$ injections $[2,15]$. Some national guideline committees refrain from giving any specific guidance on the best initiation strategy [16]. Other recommendations define the dosing scheme in relation to the drug used (according to the study protocols) [16-19]. Still other societies suggest a minimum of 4 initial injections in accordance with the DRCR.net treatment protocol [20, 21], which was also adopted in a slightly modified form in a recent head-to-head comparison of anti-VEGF drugs in the treatment of DME [9]. In the DRCR study, the majority of patients received an intensive upload ( 6 monthly treatments initially) that was not mandated, suggesting the beneficial effects of a more intense initial schedule [2], with decreased dosing requirement seen in Year 2 [15].

Aflibercept is a $115 \mathrm{kDa}$ fusion protein composed of extracellular domains from human VEGF receptors 1 and 2, and has been shown to inhibit both VEGF-A and placental growth factor (PGF) [22]. Preclinical studies have shown a long duration of action and high binding affinity to VEGF-A.

Given the variability in guidance and incomplete evidence base, it may be valuable to examine more closely the efficacy data for a well-studied initiation protocol, IAI $2 \mathrm{mg}$ every 4 weeks (2q4) for 5 doses, and in particular to ascertain if there is evidence for continued improvement following the fourth and fifth injections in the series. This post hoc analysis evaluates the visit-to-visit visual acuity and CRT responses to the initial 5 IAI given in the Phase

Table 1 Treatment initiation and continuation regimens of Phase 3 randomized controlled trial of anti-VEGF in DME

\begin{tabular}{|c|c|c|}
\hline Randomized controlled trial & Initiation and continuation regimen & Time to maximum mean BCVA* \\
\hline $\begin{array}{l}\text { RIDE/RISE } \\
\text { NCT00473382 } \\
\text { NCT00473330 }\end{array}$ & $\begin{array}{l}\text { Fixed q4 intervals ranibizumab } \\
\text { (no reduction before week } 156 \text { in the open-label extension) [4] }\end{array}$ & Week 88/week 92 \\
\hline $\begin{array}{l}\text { RESTORE } \\
\text { NCT00687804 }\end{array}$ & $\begin{array}{l}\text { Initial } 3 \text { q4 intervals followed by PRN (protocol-driven) ranibizumab retreatment } \\
{[5,6]}\end{array}$ & Week 40 \\
\hline $\begin{array}{l}\text { RELIGHT } \\
\text { NCT01257815 }\end{array}$ & Initial 3 q4 intervals followed by PRN (protocol-driven) ranibizumab retreatment [7] & Week 24 \\
\hline $\begin{array}{l}\text { REVEAL } \\
\text { NCT00989989 }\end{array}$ & Initial 3 q4 intervals followed by PRN (protocol-driven) ranibizumab retreatment [8] & Week 32 \\
\hline $\begin{array}{l}\text { DRCR protocol I } \\
\text { NCT00445003 }\end{array}$ & $\begin{array}{l}\text { Initial } 4 \text { q4 intervals ranibizumab followed by protocol-driven ranibizumab retreat- } \\
\text { ment [9] }\end{array}$ & Week 232 \\
\hline $\begin{array}{l}\text { DRCR.net protocol T } \\
\text { NCT01627249 }\end{array}$ & $\begin{array}{l}\text { Aggressive retreatment protocol for } \mid \mathrm{Al} \text {, bevacizumab, and ranibizumab prior to } \\
24 \text {-week visit with q4 intervals [10] }\end{array}$ & Week 32, week 52 \\
\hline $\begin{array}{l}\text { VIVID/VISTA } \\
\text { NCT01363440 NCT01331681 }\end{array}$ & Initial 5 q4 intervals IAI followed by 2 q8 or 2 q4 intervals [11] & Week 44 \\
\hline $\begin{array}{l}\text { BOLT } \\
\text { EUDRACT2007-000847-89 }\end{array}$ & $\begin{array}{l}\text { Initial } 3 \text { q6 intervals bevacizumab (initially combined with laser) followed by PRN } \\
\text { (protocol-driven) bevacizumab retreatment [12] }\end{array}$ & Week 100 \\
\hline
\end{tabular}

q4, every 4 weeks

* Within controlled/core study phase 
3 trials. This detailed look at the treatment response during the upload phase provides greater clarity regarding the responsiveness of DME to this upload strategy.

\section{Methods}

VISTA and VIVID were 2 similarly designed, doublemasked, randomized, active-controlled, 148-week, Phase 3 trials. VISTA was conducted across 54 sites in the United States, and VIVID took place in 73 sites across Europe, Japan, and Australia. Both VISTA and VIVID were conducted in compliance with the International Conference on Harmonisation guidelines and the Health Insurance Portability and Accountability Act of 1996. The study protocol has been described in detail [11]. Briefly, patients with central-involved DME (defined as retinal thickening involving the $1-\mathrm{mm}$ central area as measured by optical coherence tomography) were eligible for enrollment if BCVA was between 73 and 24 letters (20/40-20/320 Snellen equivalent) in the study eye. After 5 initial doses at 4-week intervals (IAI 2q4), IAI eyes received treatment in accordance with the protocol $[2 \mathrm{q} 4$, 2 mg every 8 weeks (2q8)].

This study was a post hoc analysis of the initial response to IAI treatment in the VISTA (NCT01363440) and VIVID (NCT01331681) trials, with BCVA performed per a defined protocol at every visit, OCT performed with a SD-OCT at every visit, and read by independent reading centers. Pooled data included all patients in the full analysis set from both studies, defined as all randomized patients who received any investigational product and had a baseline and at least 1 post-baseline assessment of BCVA. We evaluated the change in the BCVA and the reduction in CRT from the immediately preceding visit to the immediately following visit for each of the first 5 IAI $2 \mathrm{q} 4$ doses. This analysis was done for the pooled data from the $2 \mathrm{q} 4$ and $2 \mathrm{q} 8$ groups of VIVID and VISTA, as both groups received the same number of treatments during the upload phase. Therefore, the analysis reflects the approved dosing in both the European Medicines Agency and US Food and Drug Administration labels.

When assessing the visit-to-visit change, a threshold of 5 Early Treatment Diabetic Retinopathy Study (ETDRS) letters and $30 \mu \mathrm{m}$ was chosen as the cutoff for defining categorical change (for the responder analysis). These cutoff values were chosen, as lower thresholds would be more vulnerable to measurement variability. The change in retinal thickness of $30 \mu \mathrm{m}$ corresponds to approximately one-tenth of the total thickness. The proportion of gainers and losers and the ratio of gainers/losers were calculated for each of the first 5 dosing intervals.

In addition, as a secondary analysis, the proportion of patients who gained $\geq 5$ ETDRS letters from baseline was compared between the pooled $2 \mathrm{q} 8$ and the pooled $2 \mathrm{q} 4$ treatment arms of VIVID and VISTA by for each followup visit through week 72 . Finally, we summarized the 100 -week safety data for these 2 pooled treatment groups in order to provide a more complete comparison.

\section{Results \\ Upload analysis}

There were 576 patients in the pooled full analysis set. The highest percentage of patients attaining visual gain of at least 5 letters was seen 4 weeks after the first IAI (59.8 \%, Fig. 1). The lowest percentage of patients experiencing visual acuity loss of at least 5 letters was also seen 4 weeks after the first IAI (2.6 \%, Fig. 1). At 4 weeks following the fifth IAI, $14.4 \%$ of patients ( 1 in 7 ) had BCVA gain $\geq 5$ letters, while $8.0 \%$ had BCVA loss of $\geq 5$ letters (Fig. 1). The ratio of gainers to losers after the first IAI was 23.0; it decreased to 2.7 after the second IAI and remained around 2 subsequently $(2.3,1.7$, and 1.8 after the third, fourth and fifth IAI, respectively). Thus there were more gainers than losers following all 5 treatments of initial 2q4 dosing (Figs. 1, 2).

The anatomic response of these patients was supported by the CRT measurements showing similar proportions of reductions and increases (Fig. 3). The highest percentage of patients attaining reduction in CRT of $\geq 30 \mu \mathrm{m}$ was seen 4 weeks after the first IAI $(76.8 \%$, Fig. 3$)$, as was the lowest percentage of patients with CRT increase (2.6\%). At 4 weeks following the fifth IAI, $12.0 \%$ of patients (about 1 in 8 ) had CRT reduction of $\geq 30 \mu \mathrm{m}$ whereas $4.9 \%$ had CRT increase of $\geq 30 \mu \mathrm{m}$. Thus, the ratio of gainers to losers after the first IAI was 29.5; it decreased after each subsequent IAI but was still 2.4 following the fifth IAI (Fig. 3).

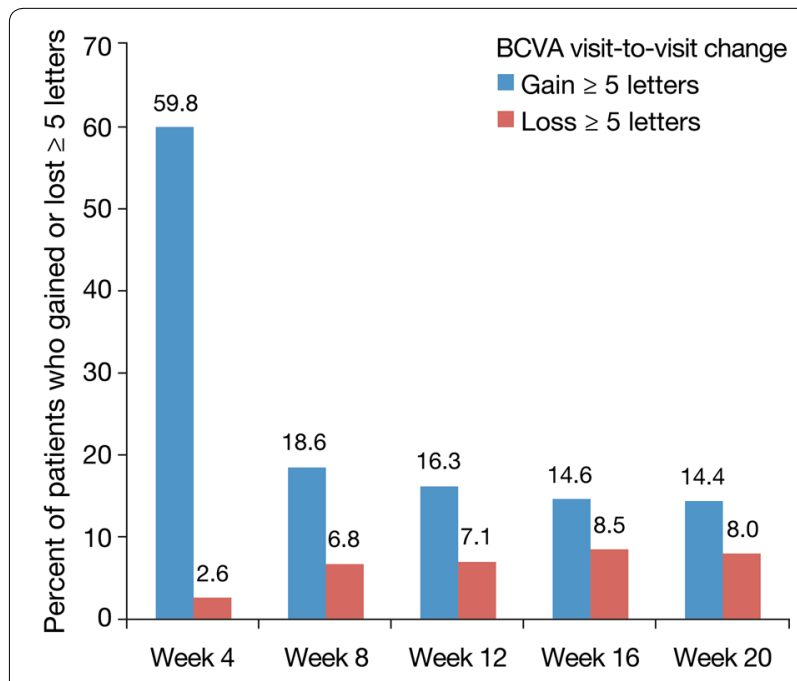

Fig. 1 Percentage of patients who gained or lost 5 letters; change of BCVA is always compared with the previous visit $(n=576)$ 


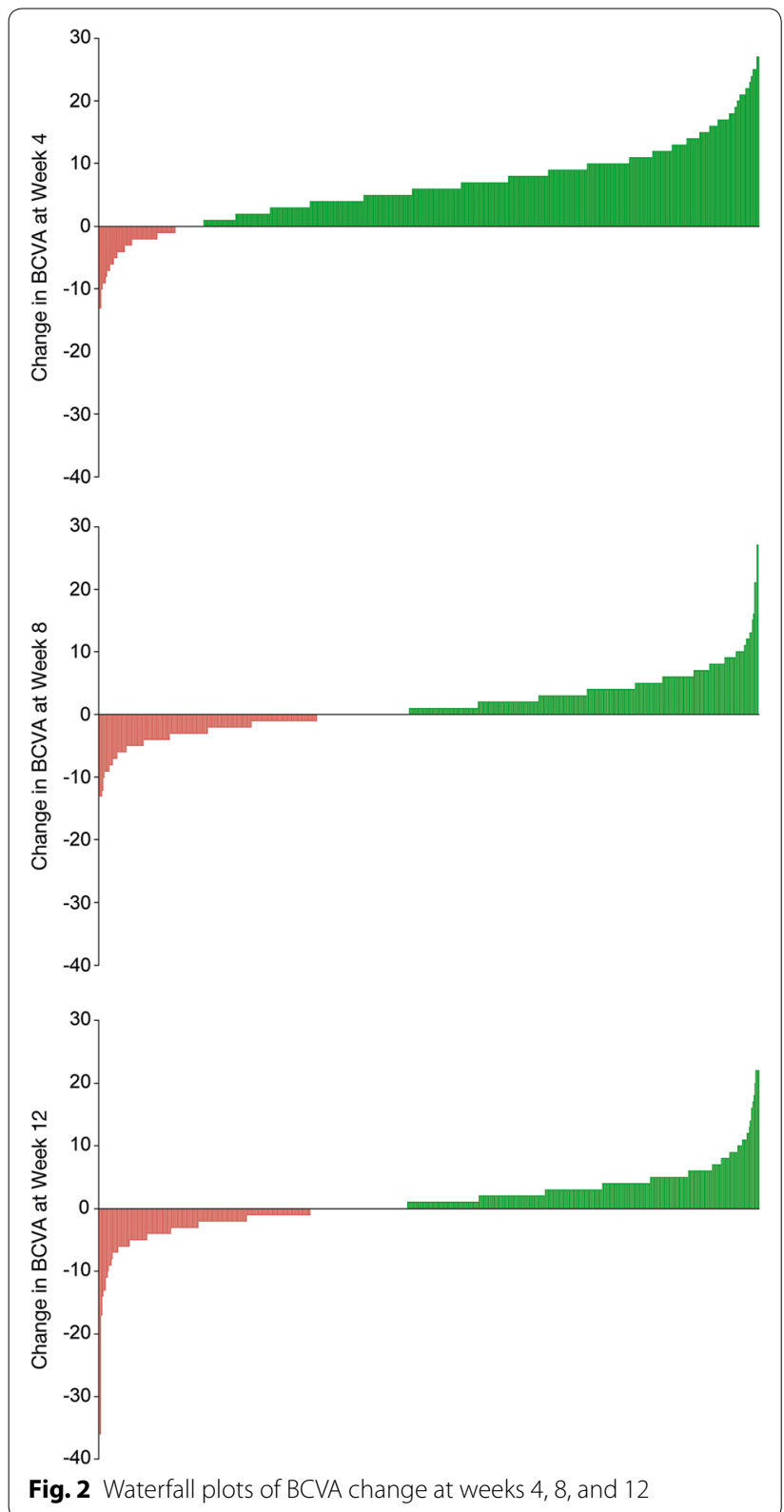

\section{Secondary analyses}

The mean change in BCVA from baseline to each visit up to 72 weeks for the pooled $2 q 8$ and the pooled $2 q 4$ arms of VIVID and VISTA is shown in Fig. 4. There is continuous improvement through week 20, representing the 4-week follow-up following the fifth IAI, with $75.8 \%$ of patients in the $2 \mathrm{q} 4$ and $79.8 \%$ of patients in the 2 q8 groups gaining $\geq 5$ letters at week 20 . During further treatment for 1 year, during which the $2 q 4$ group received treatment every 4 weeks and the 2 q8 group received treatment every 8 weeks, no large shifts of these proportions were observed for either dosage arm and the values were similar at each time point.

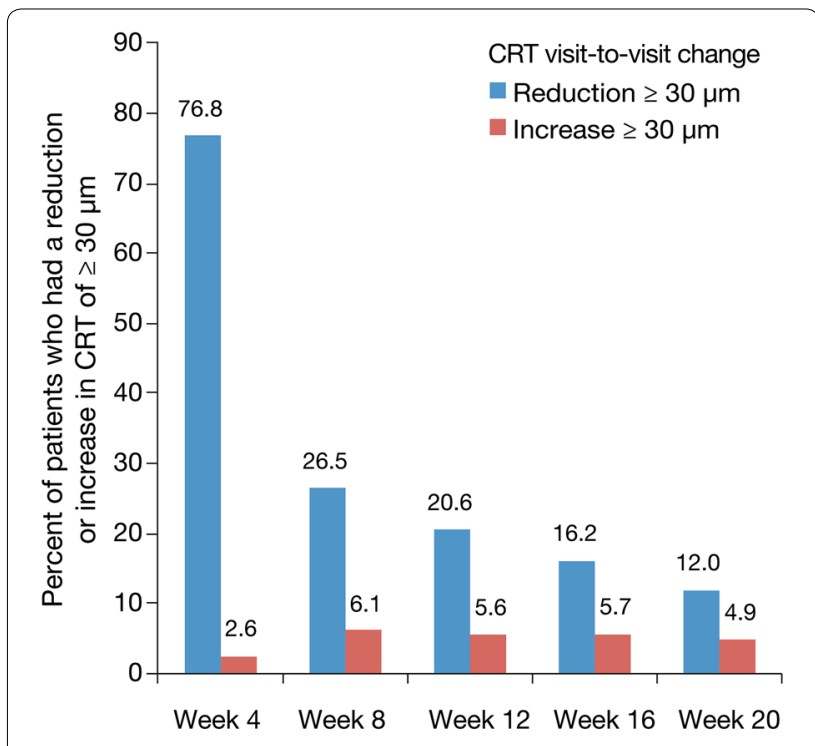

Fig. 3 Visit-to-visit change in CRT: percentage of patients who had a reduction or increase in CRT of $\geq 30 \mu \mathrm{m}(n=576)$

\section{Safety}

In both VISTA and VIVID, overall incidence of ocular and nonocular adverse events (AEs) was similar across treatment groups [11]. Over the course of the 100-week treatment period, there were no cases of endophthalmitis in any eyes treated with IAI in either study. The most common ocular AEs were conjunctival hemorrhage [99/291 (34.0 \%) IAI 2q4, 81/287 (28.2 \%) IAI 2q8, and 180/578 (31.1\%) all IAI], cataract [36 (12.4\%), $31(10.8 \%), 67(11.6 \%)]$, and eye pain [34 (11.7\%), 28 (9.8 \%), 62 (10.7 \%)]. The most common non-ocular AEs, respectively, were hypertension [67 (23.0\%), 63 (22.0\%), $130(22.5 \%)$ ], nasopharyngitis [49 (16.8\%), 51 (17.8\%), $100(17.3 \%)$ ], and urinary tract infection [23 (7.9\%), 36 (12.5\%), 59 (10.2 \%)] [11]. Overall rates of Anti-Platelet Trialists' Collaboration-defined arterial thromboembolic events, including vascular deaths, were low and comparable across the treatment groups. The incidence of death in the IAI $2 \mathrm{q} 4$, IAI $2 \mathrm{q} 8$, and control groups was 5.2, 2.6, and $1.9 \%$, respectively, in VISTA, and $2.9,4.4$, and $0.8 \%$ in VIVID [11]. Overall, the AE profile over the 100 weeks of the study was consistent with the known safety profile of IAI.

\section{Discussion}

This analysis illustrates the dynamic nature of the improvements in visual acuity during the upload phase, or first 20 weeks, of IAI $2 \mathrm{q} 4$ treatment. Specifically, in this analysis of pooled VIVID/VISTA data, we have shown that even though most shifts toward better visual acuity already occur after the very first injection, there 


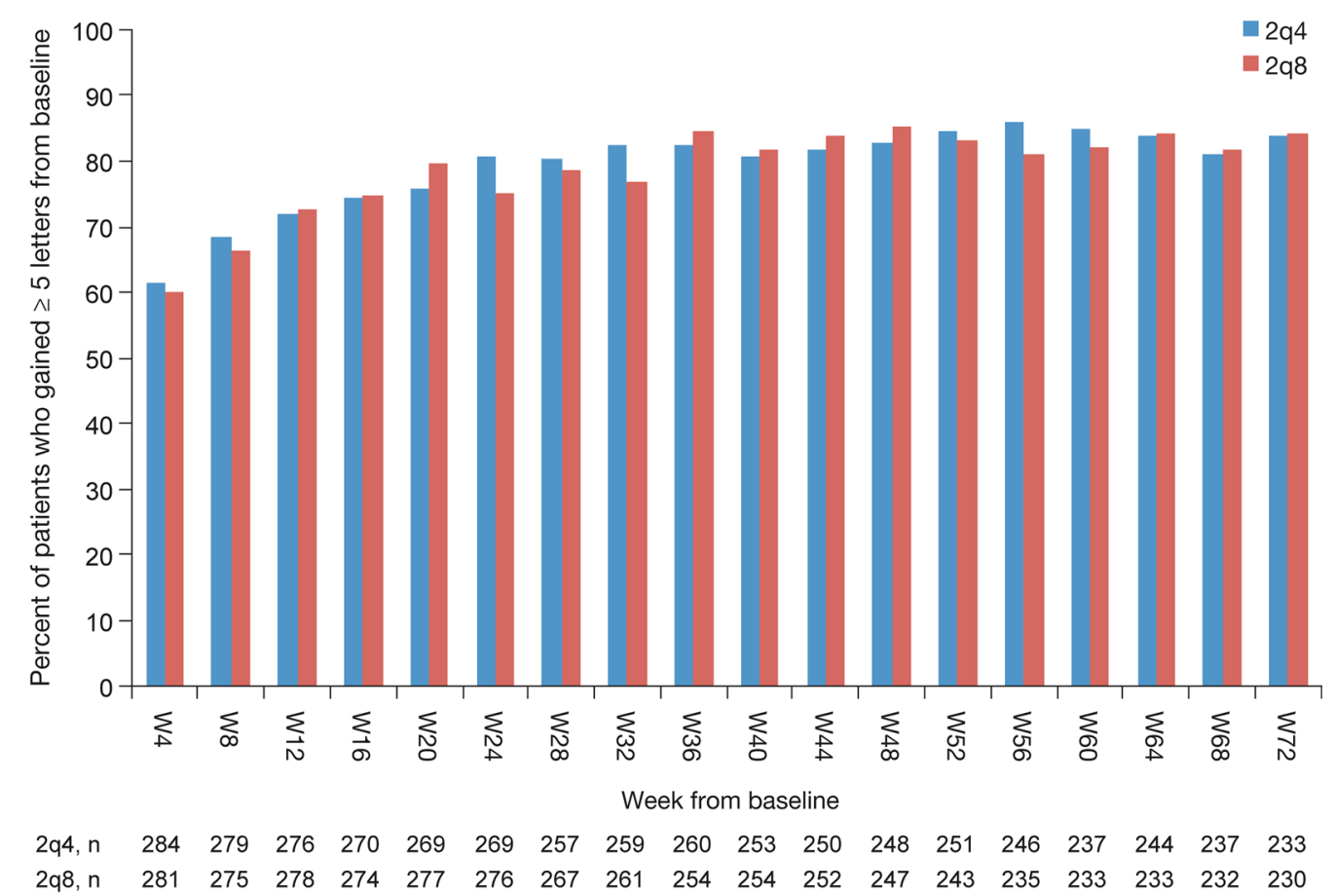

Fig. 4 Percentage of patients who gained $\geq 5$ letters from baseline, by visit

are still considerably more BCVA gainers than losers following each of the subsequent 4 treatments during the IAI 2 q4 upload phase. This response in vision of patients with DME is paralleled by the CRT measurements showing similar proportions of reductions and increases. Therefore, these data support the potential benefit of at least 5 initial IAI $2 \mathrm{q} 4$ as an upload treatment regimen.

We also showed that over the course of the subsequent 52 weeks, during which one treatment arm received IAI $2 \mathrm{q} 8$ and the other continued to receive IAI $2 \mathrm{q} 4$, the visual acuity efficacy results, as well as the safety results, were highly similar. These data indicate that the longer-term outcome appears to be similar with less frequent dosing after the upload phase.

The data presented here are consistent with what has been observed in other important studies of anti-VEGF treatment for DME (Table 1). As these studies have shown, the rise in visual acuity in DME is gradual and peak visual acuity is only established after $6-9$ months or longer [23]. This suggests that the initial treatment phase is particularly critical for final treatment success [10, 24, 25]. Although caution must be exercised when comparing across studies, there seems to be a pattern demonstrated in studies with a less intense/rigorous treatment initiation that outcomes may not be as good as outcomes in studies with a more rigorous and intense initiation scheme (e.g., RESTORE, Protocol I) [5, 6, 10].
With regard to IAI, the Phase 2 DA VINCI study showed for the first time that outcomes are similar with anIAI 2q8 dosing schedule compared with $2 \mathrm{q} 4$ or PRN dosing, and the requirement for monitoring can be reduced [13]. However, the study also suggests that visual acuity is less stable in the early treatment phase and that a more intensified treatment initiation could be beneficial. Hence, in the Phase 3 studies for IAI, an additional dose was added at week 12, resulting in a sequence of 5 initial IAI $2 \mathrm{q} 4$ before extending the treatment interval to $2 q 8$ in one of the treatment arms. In summary, the available data suggest that visual acuity in DME patients may be impacted by the number of initial treatments during the upload phase. Our analysis of the pooled data from the upload phase of VIVID and VISTA suggests that this vulnerable phase may best be managed with the intensified dosing regimen of 5 initial consecutive doses at 4-weeks intervals.

The implementation of an initial treatment series can minimize the risk of under-treatment. In particular for patient education, the concept of a consistent initiation series can enhance the attendance at follow-up visits and adherence to the therapy [26]. If patients understand the need for a strict retreatment, the chance of reaching the maximum BCVA potential may be higher. A series of treatments can reduce organizational efforts and might minimize the impact of potential diagnostic 
errors (optical coherence tomography criteria) at decision points. Moreover, the administration of 5 consecutive IAIs does not preclude any additional interim fundus or safety examination, if considered necessary on a caseby-case basis.

This analysis has important strengths. First, it is based on a large data set from 2 pivotal randomized controlled trials of patients treated with the identical upload regimen. Second, it fills a gap in the field insofar as this particular regimen has not been well represented in previous analyses. Third, longer-term efficacy and safety strengthen the finding that following this particular upload regimen, outcomes remain similar despite less frequent IAI dosing (2q8 vs. 2q4).

This analysis also has several limitations. Only 1 loading dose phase is analyzed; thus, no direct conclusions can be drawn about the comparative efficacy of this initial treatment schedule with any other treatment scheme. In addition, the duration of action of each injection is not necessarily limited to the first 4 weeks following the injection, as the long-term experience suggests a vascular remodeling in DME under anti-VEGF therapy [9]. Thus, the visit-to-visit change cannot be solely attributedto the most recent injection, but also to the previous treatment, as well asother confounding factors. Although the simple responder analysis of the visit-to-visit change, as carried out in this study and described in this article, does not allow for predicting or assessing the response of an individual patient, it is supportive of an intensive treatment start, which is in line with observations that have been made in other studies.

\section{Conclusions}

This post hoc analysis has demonstrated continuous improvement during the treatment initiation schedule of 5 IAI at 4-weeks intervals implemented in the VIVID and VISTA randomized clinical trials. These data support the potential benefit of this pragmatic treatment initiation strategy in the treatment of DME.

\begin{abstract}
Abbreviations
2q4: $2 \mathrm{mg}$ given every 4 weeks (intravitreal aflibercept injections); 2 q8: $2 \mathrm{mg}$ every 8 weeks (intravitreal aflibercept injections); BCVA: best-corrected visual acuity; CRT: central retinal thickness; DME: diabetic macular edema; ETDRS: Early Treatment Diabetic Retinopathy Study; IAl: intravitreal aflibercept injection; OCT: optical coherence tomography; SD-OCT: spectral-domain optical coherence tomography; VEGF: vascular endothelial growth factor.
\end{abstract}

\section{Authors' contributions}

FZ: Research design. FZ: Data collection. FZ, PGS, JIL, HA, GEL, FB: Analysis and interpretation. FZ, PGS, JIL, HA, GEL, FB: Manuscript preparation. All authors read and approved the final manuscript.

\section{Authors' information}

F.Z. is spokesman of the task force'seeing in old age' of the German organization of blind and partially sighted people (DBSV) and has been the Principal
Investigator in several clinical trials, with a special focus on healthcare research. He is Vice Chair of the ad-hoc committee of the early benefit assessment for the German Professional Medical Societies and supports the team of the macula committee, establishing the clinical guidelines of the German scientific societies, in particular regarding DME treatment. P.G.S. is the Director of Ophthalmology at the Organizacion Medica de Investigacion in Buenos Aires, Argentina. Specializing in diabetic macular edema and retinal vein occlusion, he has been the Principal Investigator for a number of clinical trials investigating anti-VEGF treatment in $\mathrm{AMMD}$ and DME, including RIDE, VIEW2, SAKURA and OCTAVE. J.I.L. is the Marion H. Schenk, Esq., Chair in Ophthalmology for Research of the Aging Eye, Professor of Ophthalmology, and Director, Retina Service at the University of Illinois College of Medicine at Chicago. She is also the President of the Chinese American Ophthalmology Society and Chairs both the Retina Society Credentialing Committee and Macula Society Research and Education Committee. She is a leading authority on retina and vitreoretinal diseases, directing more than 50 clinical research studies and trials on age-related macular degeneration, diabetic retinopathy, macular edema and retinal vein occlusion and complex retinal detachments and generating more than 220 peer-reviewed journal articles. H.A. is the Deputy Director and Head of the Retina Section at the Eye Center of the University Hospital, University of Freiburg, Germany. He is member of the board of the German Retinological Society and leading an active research group with a special focus on angiogenesis. G.E.L. is Professor of Ophthalmology at the Department of Ophthalmology at the University Eye Hospital Ulm, Ulm, Germany. She is Director of the Section of Medical Retina and Laser Treatment. Professor Lang's major clinical interest is diagnosis and treatment of retinal diseases. Her major research fields are preclinical and clinical studies concerning diabetic retinopathy, retinal vein occlusions, and AMD. She is coordination investigator, principal investigator and investigator in several clinical trials and member of advisory boards and steering committees. She is former President of the German Society of Ophthalmology, former President of the German Initiative Group of Early Detection of Diabetic Eye Disease (IFDA) and former Vice President of the German Retinological Society. She has authored and co-authored over 190 articles in peer-reviewed journals and has published several books. Since 2002, she is chief editor of "Klinische Monatsblätter für Augenheilkunde". F.B. is Professor at the Scientific University Institute San Raffaele, Milan, Italy. $\mathrm{He}$ is President of the Academia Ophthalmologica Europea, Past-President of EURETINA, and Past-Vice-President of EuroLam. He is also Co-Editor of the European Journal of Ophthalmology and a former board member of the Jules Gonin Club, Macula Society, and EVER. He is a member of the Academia Ophthalmologica Internationalisa and Accademia Nazionale di Medicina. Professor Bandello has authored or co-authored over 338 medical articles, which are mainly related to retinal diseases.

\section{Author details \\ ${ }^{1}$ Centre for Ophthalmology, Eberhard-Karls University of Tuebingen, Schleich- strasse 12, 72076 Tübingen, Germany. ${ }^{2}$ Organizacion Medica de Investigacion, Uruguay 725 PB, C1015ABO Buenos Aires, Argentina. ${ }^{3}$ University of Illinois at Chicago, Illinois Eye and Ear Infirmary, 1855 W. Taylor Street, M/C 648, Chicago, IL 60612, USA. ${ }^{4}$ Eye Center, Medical Center, Faculty of Medicine, University of Freiburg, Killianstr. 5, 79106 Freiburg, Germany. ${ }^{5}$ Division of Medi- cal Retina and Laser Surgery, Department of Ophthalmology, University Eye Hospital Ulm, Prittwitzstr. 43, 89075 Ulm, Germany. ${ }^{6}$ Department of Oph- thalmology, IRCCS San Raffaele Scientific Institute, University Vita-Salute San Raffaele, Via Olgettina 60, 20132 Milan, Italy.}

\section{Acknowledgements}

Editorial assistance (proofreading and minor editing) was provided by Bill Kadish, MD, and Albert M. Balkiewicz, MSc. This supporting activity of PAREXEL was funded by Bayer Pharmaceuticals.

\section{Competing interests}

The author(s) have made the following disclosures: F.Z. has received consulting fees from Alimera, Allergan, Bayer Pharmaceuticals, and Novartis, and speaker fees from Alcon, Alimera, Allergan, Bayer Pharmaceuticals, Heidelberg Engineering, and Novartis. P.G.S. is an advisor to Novartis, Roche/Genentech, Bayer, and Allergan, as well as a speaker for Novartis, Roche/Genentech, and Bayer. He has also received travel grants from Novartis. J.I.L. has been the principal investigator for the DRCR Protocol I, serves on DMCs for Santen and received honoraria from Regeneron, Genentech, Alcon, Lumenis, AbbVie, Hospira/Pfizer and Icon Bioscience within the last 12 months. H.A. has received 
consulting fees from Novartis and Bayer Pharmaceuticals, and speaker fees from Allergan, Bayer Pharmaceuticals, Zeiss, and Novartis. G.E.L. reports the following conflicts of interest: Novartis Pharma GmbH (financial support, consultant, recipient), Alcon Pharma GmbH (financial support), Allergan (recipient), Carl Zeiss Meditec AG (financial support, recipient), Bayer Pharmaceuticals (consultant, recipient), Boehringer Ingelheim Pharma GmbH \& Co. KG (financial support, consultant, recipient). F.B. is an Advisory board member for Alcon, Alimera, Allergan, Bausch and Lomb, Bayer, Genentech, Novagali Pharma, Novartis, Pfizer, Roche, Sanofi-Aventis, Sifi, Sooft, Théa, Thrombogenics, and Zeiss.

\section{Availability of data and materials}

The dataset(s) supporting the conclusions of this article are not available for sharing; the data included in this manuscript is not in the current scope of clinical trial data sharing according to Bayer's transparency policy. This scope was carefully defined based on the EFPIA and PhRMA principles on responsible clinical trial data sharing.

\section{Consent for publication}

This analysis does not include any personal information or image. All patients provided informed consent to the scientific analysis and publication of the anonymized data.

\section{Ethics approval and consent to participate}

Each clinical site's respective institutional review board/ethics committee approved the respective original studies. All patients provided written informed consent. Both VISTA and VIVID were conducted in compliance with the International Conference on Harmonization guidelines and the Health Insurance Portability and Accountability Act of 1996.

\section{Funding}

The original studies (VISTA and VIVID) were funded by Regeneron Pharmaceuticals, Inc, Tarrytown, New York, and Bayer Pharmaceuticals, Berlin, Germany. The sponsors participated in the design and conduct of the original studies and analysis of the data.

Received: 30 April 2016 Accepted: 15 June 2016

Published online: 11 July 2016

\section{References}

1. Heier JS, Bressler NM, Avery RL, Bakri SJ, Boyer DS, Brown DM, et al. Comparison of aflibercept, bevacizumab, and ranibizumab for treatment of diabetic macular edema: extrapolation of data to clinical practice. JAMA Ophthalmol. 2016:134:95-9.

2. Korobelnik JF, Kleijnen J, Lang SH, Birnie R, Leadley RM, Misso K, et al. Systematic review and mixed treatment comparison of intravitreal aflibercept with other therapies for diabetic macular edema (DME). BMC Ophthalmol. 2015;15:52.

3. Virgili G, Parravano M, Menchini F, Evans JR. Anti-vascular endothelial growth factor for diabetic macular oedema. Cochrane Database Syst Rev. 2014;10:007419.

4. Boyer DS, Nguyen QD, Brown DM, Basu K, Ehrlich JS. Outcomes with as-needed ranibizumab after initial monthly therapy: longterm outcomes of the phase III RIDE and RISE trials. Ophthalmology. 2015;122:2504-13.

5. Schmidt-Erfurth U, Lang GE, Holz FG, Schlingemann RO, Lanzetta P, Mas$\sin \mathrm{P}$, et al. Three-year outcomes of individualized ranibizumab treatment in patients with diabetic macular edema: the RESTORE extension study. Ophthalmology. 2014;121:1045-53.

6. Mitchell P, Massin P, Bressler S, Coon CD, Petrillo J, Ferreira A, et al. Threeyear patient-reported visual function outcomes in diabetic macular edema managed with ranibizumab: the RESTORE extension study. Curr Med Res Opin. 2015;31:1967-75.

7. Pearce I, Banerjee S, Burton BJ, Chakravarthy U, Downey L, Gale RP, et al. Ranibizumab 0.5 mg for diabetic macular edema with bimonthly monitoring after a phase of initial treatment: 18-month, multicenter, phase IIIb RELIGHT study. Ophthalmology. 2015;122:1811-9.
8. Ishibashi T, Li X, Koh A, Lai TY, Lee FL, Lee WK, et al. The REVEAL study: ranibizumab monotherapy or combined with laser versus laser monotherapy in Asian patients with diabetic macular edema. Ophthalmology. 2015;122:1402-15.

9. Bressler SB, Glassman AR, Almukhtar T, Bressler NM, Ferris FL, Googe JM $\mathrm{Jr}$, et al. Five-year outcomes of ranibizumab with prompt or deferred laser versus laser or triamcinolone plus deferred ranibizumab for diabetic macular edema. Am J Ophthalmol. 2016;164:57-68.

10. Wells JA, Glassman AR, Ayala AR, Jampol LM, Aiello LP, Antoszyk AN, et al. Aflibercept, bevacizumab, or ranibizumab for diabetic macular edema. $N$ Engl J Med. 2015;372:1193-203.

11. Brown DM, Schmidt-Erfurth U, Do DV, Holz FG, Boyer DS, Midena E, et al. Intravitreal aflibercept for diabetic macular edema: 100-week results from the VISTA and VIVID studies. Ophthalmology. 2015;122:2044-52.

12. Rajendram R, Fraser-Bell S, Kaines A, Michaelides M, Hamilton RD, Esposti SD, et al. A 2-year prospective randomized controlled trial of intravitreal bevacizumab or laser therapy (BOLT) in the management of diabetic macular edema: 24-month data: report 3. Arch Ophthalmol. 2012;130:972-9.

13. Do DV, Nguyen QD, Boyer D, Schmidt-Erfurth U, Brown DM, Vitti R, et al. One-year outcomes of the da Vinci study of VEGF trap-eye in eyes with diabetic macular edema. Ophthalmology. 2012;119:1658-65.

14. Wells JA, Glassman AR, Jampol LM, Aiello LP, Antoszyk AN, Baker CW, et al. Association of baseline visual acuity and retinal thickness with 1-year efficacy of aflibercept, bevacizumab, and ranibizumab for diabetic macular edema. JAMA Ophthalmol. 2016:134:127-34.

15. Wells JA, Glassman AR, Ayala AR, Jampol LM, Bressler NM, Bressler SB, et al. Aflibercept, bevacizumab, or ranibizumab for diabetic macular edema: two-year results from a comparative effectiveness randomized clinical trial. Ophthalmology. 2016. [Epub ahead of print].

16. Thomas BJ, Shienbaum G, Boyer DS, Flynn HW Jr. Evolving strategies in the management of diabetic macular edema: clinical trials and current management. Can J Ophthalmol. 2013;48:22-30.

17. Preferred practice pattern: diabetic retinopathy American Academy of Ophthalmology. American Academy of Ophthalmology web site. http:// www.aao.org/preferred-practice-pattern/diabetic-retinopathy-pppupdated-2016. Accessed 4 April 2016.

18. Bandello F, Cunha-Vaz J, Chong NV, Lang GE, Massin P, Mitchell P, et al. New approaches for the treatment of diabetic macular oedema: recommendations by an expert panel. Eye. 2012;26:485-93.

19. Hooper P, Boucher MC, Colleaux K, Cruess A, Greve M, Lam WC, et al. Contemporary management of diabetic retinopathy in Canada: from guidelines to algorithm guidance. Ophthalmologica. 2014;231:2-15.

20. Aiello LP, Beck RW, Bressler NM, Browning DJ, Chalam KV, Davis M, et al. Rationale for the diabetic retinopathy clinical research network treatment protocol for center-involved diabetic macular edema. Ophthalmology. 2011;118:e5-14.

21. Ziemssen F, Helbig H, Lemmen KD, Spital G, Bertram B, Hillenkamp J, et al. [Statement of the German Ophthalmological Society, the Retina Society and the Professional Association of German Ophthalmologists: treatment of diabetic maculopathy (April 2013)]. Klin Monbl Augenheilkd. 2013;230:614-28

22. Deissler HL, Lang GK, Lang GE. Capacity of aflibercept to counteract VEGF-stimulated abnormal behavior of retinal microvascular endothelial cells. Exp Eye Res. 2014;122:20-31.

23. Minami Y, Nagaoka T, Ishibazawa A, Yoshida A. Short-term effect of intravitreal ranibizumab therapy on macular edema after branch retinal vein occlusion. Retina. 2016. [Epub ahead of print].

24. Ebneter A, Wolf S, Zinkernagel MS. Prognostic significance of foveal capillary drop-out and previous panretinal photocoagulation for diabetic macular oedema treated with ranibizumab. Br J Ophthalmol. 2016;100:365-70

25. Korobelnik JF, Do DV, Schmidt-Erfurth U, Boyer DS, Holz FG, Heier JS, et al. Intravitreal aflibercept for diabetic macular edema. Ophthalmology. 2014;121:2247-54.

26. Mann DM, Ponieman D, Leventhal H, Halm EA. Predictors of adherence to diabetes medications: the role of disease and medication beliefs. J Behav Med. 2009;32:278-84. 